\title{
EFFICIENT ID BASED SECURED TROLLEY SYSTEM USING CLOUD COMPUTING
}

\author{
Mr. Mani.A \\ Associate Professor/Department of Computer Science and Engineering \\ S.A. Engineering College, Chennai 600077. \\ Aishwarya.D, Logeswari.M, Mythili.R \\ UG Student/Department of Computer Science and Engineering \\ S.A. Engineering College, Chennai 600077
}

\begin{abstract}
A shopping precinct or advanced may be a place wherever folks purchase product/s for his or her regular use. the shoppers have to be compelled to wait in long queues to induce their product scanned mistreatment Universal Product Code scanner and find it beaked. To get eliminate this, we've got planned a replacement 'Shopping self-propelled cart RFID. This process is employed to help an individual whereas looking and conjointly to avoid standing in long queues and so saving time. The individuals within the looking centers can have RFID tags to retrieve/access info concerning it. once a client places a product within the streetcar, the RFID Reader can scan the merchandise ID and also the info associated with it'll be hold on in controller. There will be communication between automaton device, main server and request system (gate system) via transmitter and receiver module and by using cloud technology we can do the business analysis and the data is stored in the cloud for further preference.
\end{abstract}

Keywords: Transmitter and receiver, RFID Technology, cloud technology, Arduino.

\section{INTRODUCTION}

Technology plays a really important role within the constitution of attribute and identity. Humans have shaped and extended themselves by virtue of technical tools and artifacts. individuals are always trying to develop technology which is able to support and fulfill their basic needs with a neater and faster way. With the growing economy, urbanization and industrialization in recent years it's made a big change within the global market. The grocery section plays a crucial part within the worldwide economy acquiring a decent position with most convenient and diverse business across the world. Now a day's purchasing and shopping at big malls is becoming a daily activity in metro cities especially on holidays and weekends. shopping precinct may be a place where people from all walks of life will get there daily necessities. There are some problems that are currently faced by the customer. One has got to keep worrying about the number brought whether it'll suffice the requirement or not.

The current scenario is that sometimes an individual comes with an inventory of required products, while shopping he selects the merchandise among different brands depending upon details he get after reading on the pack or the feedback. When all required products are placed into cart the person moves towards billing counter where he may need to sub a protracted queue to pay the bill depending upon the frenzy in mall, unnecessarily wasting valuable time. the frenzy is even more during the special offers and discount; during this case lots of individuals leave the mall without shopping. Some methods are incorporated to resolve the issues but it still needs an excellent improvement. there's have to bring some advancement and automation during this on going practice so everyone can feel easy and comfy while shopping whether or not there's huge rush. Increasing rate in number of malls over the years throughout the world thanks to the demand of products by the general public is considerable. Compared to some foreign countries 'shopping mall system', there's still many area where improvement are often worn out terms of providing quality shopping experience to customer.

The main problem with the prevailing system of barcode billing is that the proven fact that each product is scanned just one at a time so total scanning time grows gradually when there are many purchased products. The barcode scanner is proscribed by direct visual contact with barcodes [2]. Thus, it cannot scan barcodes that stay out of its vision. Therefore, people still tend to line up in queues ahead of cashier's desk thanks to the lack of cashier's billing speed to catch up with the flow of latest customers willing to check-out. This paper 
contributes to the advancement within the existing shopping system which may bring a replacement innovation within the field of shopping malls. the most purpose of this paper is to produce centralized and automatic billing system using RFID and RF communication. together with the automated billing some special features incorporated are budget setting Unlike barcode system, smart cart doesn't need any visual contact with barcodes which can get distorted in real world situations. All data about purchased products and user account data are stored in an exceedingly cloud database within the Internet.

Then, smart cart shows this information to customers on its display. A customer can delete an item from the list whenever he or she wishes. If the customer decides to end purchasing, just one button press is required to upload all purchased product data and their total cost to cloud database. Once all payment data is uploaded to the net, total cost is withdrawn from the registered account cash of the customer through cloud database

\section{BACKGROUND AND LITERATUR REVIEW}

In recent behaviour humans most important vital role is being played in the super markets and shopping complex. It is the primary need of every person's need. However the reality is that in normal shopping method is the monotonous way of shopping by purchasing the products waiting in a queue for a long time that make the customer tired and irritated of shopping. To over come this, we have used some technologies that can make the people to do a comfortable and easy way of shopping. The technologies to improve these technologies are:

\section{A. RFID Technology}

RFID is an acronym for "radio-frequency identification" and refers to a technology whereby digital data encoded in RFID tags or smart labels (defined below) are captured by a reader via radio waves. RFID is comparable to barcoding therein data from a tag or label are captured by a tool that stores the information during a database. RFID, however, has several advantages over systems that use barcode asset tracking software. the foremost notable is that RFID tag data will be read outside the line-of-sight, whereas barcodes must be aligned with an optical scanner. RFID belongs to a gaggle of technologies remarked as Automatic Identification and Data Capture (AIDC). AIDC methods automatically identify objects, collect data about them, and enter those data directly into computer systems with little or no human intervention. RFID methods utilize radio waves to accomplish this. At a straightforward level, RFID systems encompass three components: an RFID tag or smart label, an
RFID reader, and an antenna. RFID tags contain an microcircuit and an antenna, which are wont to transmit data to the RFID reader (also called an interrogator). The reader then converts the radio waves to a more usable type of data. Information collected from the tags is then transferred through a communications interface to a number computing system, where the info is stored during a database and analyzed at a later time.

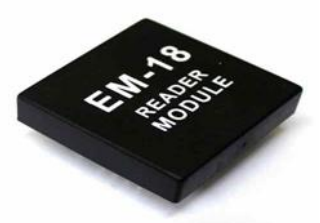

Fig 1:RFID Reader

\section{B. RFID TAG}

RFID tag consists of a microcircuit and an antenna. The tag is additionally composed of a protective material that holds the pieces together and shields them from various environmental conditions. The protective material depends on the applying. as an example, employee ID badges containing RFID tags are typically made of durable plastic, and therefore the tag is embedded between the layers of plastic. RFID tags are available in a range of shapes and sizes and are either passive or active. Passive tags are the foremost widely used, as they're smaller and fewer expensive to implement. Passive tags must be "powered up" by the RFID reader before they will be transmit data. Unlike passive tags, active RFID tags have an onboard power supply (e.g., a battery), thereby enabling them to transmit data in the least times. For a more detailed discussion, see this article: Passive RFID Tags vs. Active RFID Tags. Smart labels differ from RFID tags therein they incorporate both RFID and barcode technologies. They're product of an adhesive label embedded with an RFID tag inlay, and that they may additionally feature a barcode and/or other printed information. Smart labels are often encoded and printed ondemand using desktop label printers, whereas programming RFID tags are longer consuming and requires more advanced equipment.

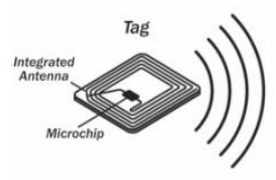

Fig 2:RFID Tag 


\section{LCD Display}

A liquid-crystal display (LCD) can be a flat-panel display or other electronically modulated device that uses the light-modulating properties of liquid crystals combined with polarizers. Liquid crystals don't emit light directly, instead employing a backlight or reflector to produce images in color or monochrome. [1] LCDs are available to display arbitrary images (as during a general-purpose computer display) or fixed images with low information content, which can be displayed or hidden, like preset words, digits, and seven-segment displays, as during a digital clock. They use the identical basic technology, except that arbitrary images are made from a matrix of small pixels, while other displays have larger elements. LCDs can either be normally on (positive) or off (negative), counting on the polarizer arrangement. for example, a personality positive LCD with a backlight will have black lettering on a background that is the colour of the backlight, and a personality negative LCD will have a black background with the letters being of the identical color because the backlight. Optical filters are added to white on blue LCDs to convey them their characteristic appearance. LCDs are employed in an exceedingly large range of applications, including LCD televisions, computer monitors, instrument panels, aircraft cockpit displays, and indoor and outdoor signage.

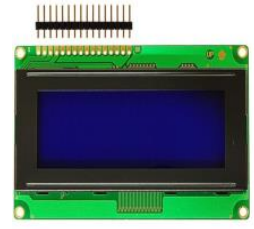

Fig 3:LCD Display

\section{Arduino UNO}

As the main processing unit of smart cart hardware is an Arduino Uno is an open-source microcontroller board supported the Microchip ATmega328P microcontroller and developed by Arduino.cc. The board is supplied with sets of digital and analog input/output pins that will be interfaced to varied expansion boards and other circuits. The Arduino Uno could be a microcontroller board supported the ATmega328 (datasheet). it's 14 digital input/output pins (of which 6 will be used as PWM outputs), 6 analog inputs, a $16 \mathrm{MHz}$ ceramic resonator, a USB connection, an influence jack, an ICSP header, and a push. It contains everything needed to support the microcontroller; simply connect it to a computer with a USB cable or power it with a AC-to-DC adapter or battery to induce started.

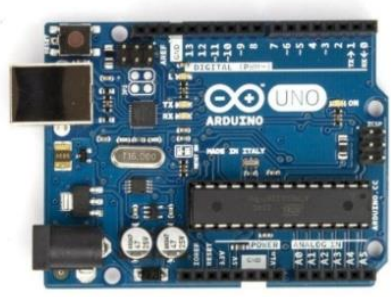

Fig 4:Arduino UNO

\section{E. Cloud Part}

The cloud could be a huge, interconnected network of powerful servers that performs services for businesses and for people. Activities like storage and processing happen within the cloud instead of on the device itself, this has had significant implications for IOT. Many IoT systems make use of enormous number of sensors to gather data and so make intelligent decisions. Using the cloud is very important for aggregating data and drawing insights from that data. for example in our project, smart trolley system cloud is employed to store the small print of the Registered customer, Product details, charge account credit details, Shop details. The Registered customers details includes cutomer_id,customer_name,

total_number_of_visits,total_amount. Product details includes Product_code, P_name, Price, Mf_date, Exp_date, Tag_id. Smartcard details includes card_id, cust_id, balance. Cloud storage involves a minimum of one data server that auser connects to via the web. The customer sends product details via RFID over the web to the data server which forwards the knowledge to multiple servers. The stored data is then accessible through a webbased interface. Cloud storage systems involve vast numbers of knowledge servers to ensure their availability. That way, if one server requires maintenance or fails, the user can rest assured that the information has been replicated elsewhere to confirm availability. for instance, the Amazon AWSCloudspans55

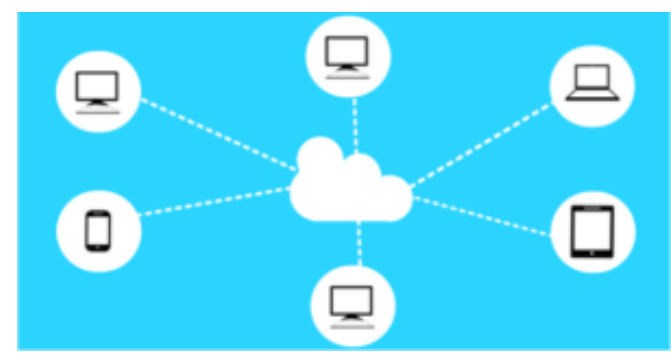

Fig 5:Cloud Storage

\section{F. Similar Project}

Ankush Yewatkar, Faiz Inamdar, Raj Singh, Ayushya, Anmol Bandal, "Smart Cart with 
Automatic Billing, Product Information, Product Recommendation Using RFID \& Zigbee with AntiTheft.", 7th International Conference on Communication, Computing and Virtualization 2016[15] proposed a smart shopping cart system that monitors bought items and furthermore online transactions for billing utilizing RFID and ZigBee. The framework will likewise give recommendations for products to purchase based on customer's purchase history from a centralized system. In this system, every item in Mart will have RFID tag, and each trolley will have RFID Reader and ZigBee joined to it. There will be a centralized system for the recommendation and online transaction as well. In addition, also there will be RFID reader at the exit of the mall for hostile to burglary.

\section{METHODOLOGY}

In general, a market will have many Smart Carts which will serve a swarm of holiday makers. of these Smart Carts hook up with the net through their WiFi modules. Firstly, the market visitor who intends to use Smart Cart system registers and creates an account within the website provided by the market. After that, this person transfers some money into this account via internet banking or other sources. Therefore the smart cart user get his purchasessithe value of his/her money and purchasee information together with the acquisition history are going to be stored within the cloud database. After creating account while the person start to get he enters the trolley number to point that the trolley is being employed. After that, the visitor does shopping, chooses items with RFID tags and swipes these tags over the RFID reader antenna of the smart cart. Therefore, smart cart gets unique ID codes of every product lying inside it and transmits these codes to the Cloud database, after which, retrieves all information associated with these things. Smart cart hardware shows the data like item names and costs on its built-in LCD screen. The customer looks into the display and may see the list of products lying inside the cart and every one relevant information and total cost so customer can make a higher decision of what to get. Customer can easily remove an unwanted product out of the cart. If the customer decides to complete purchasing and go billing, then he or she just presses the "Buzzer" button on the smart cart which can send all purchased product information through Cloud database and sense the data to the customer and therefore the shopkeeper through Wi-Fi. Shopkeeper will withdraw total cost from the private cash of the user and therefore the payments with the main points are going to be done through cloud.

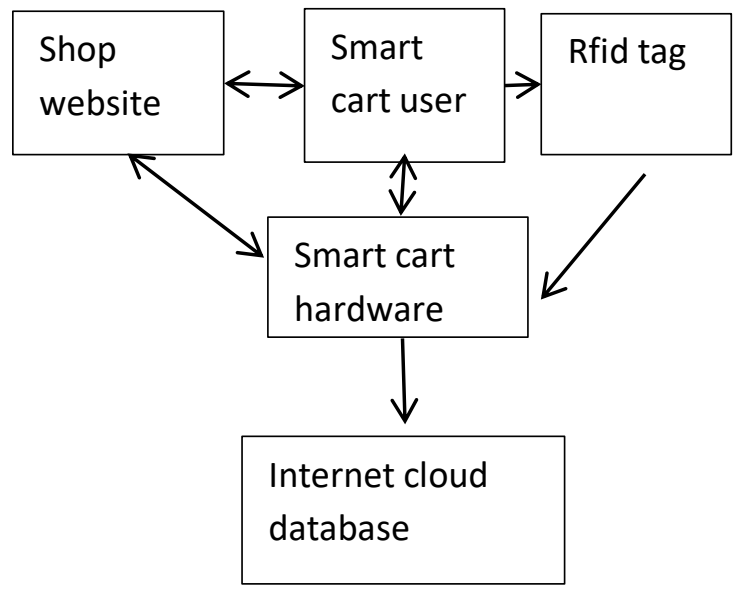

Fig 6: Block diagram of the entire system

Moreover, there are some basic steps that customer should do to accomplish the shopping process. A flowchart describing what a customer is expected to do is illustrated

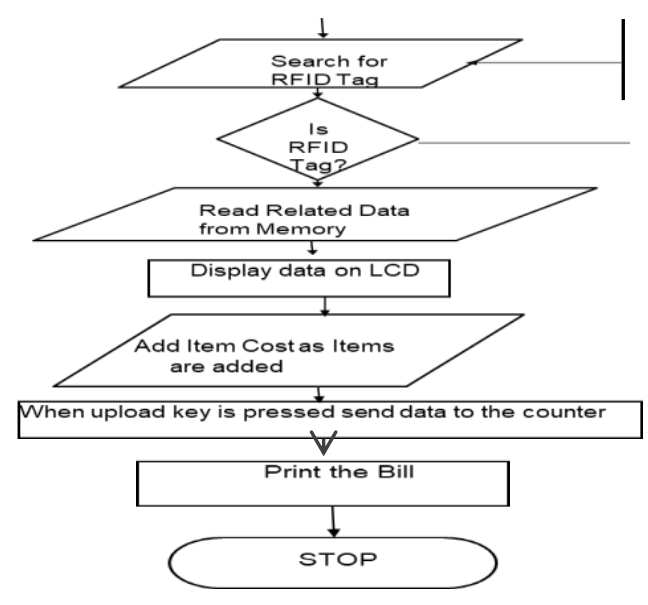

Fig 7:Flowchart

The main functions of the market website are:

1. Provide convenient visual interface and user account

2. Communicate with DropBox Cloudant and retrieve user data

3. Provide reliable money transfer from user bank accounts or web-money sources. 


\section{International Journal of Engineering Applied Sciences and Technology, 2020 \\ Vol. 4, Issue 11, ISSN No. 2455-2143, Pages 474-477 \\ Published Online March 2020 in IJEAST (http://www.ijeast.com)}

4. It provide purchase history where purchased items are recorded for a brief (or long) period of your time.

The development of the web site is that the web site won't have an instantaneous communication with the samrt card hardware. Instead, it'll retrieve the user cash balance and buy history from the Cloudant database by sending HTTP GET requests. The RESTful API working within the Cloudant server will challenge the specified data and encapsulated in HTTP protocol. It may be seen that each one data in dropbox cloud database is stored. which is extremely convenient way of representing structured key value pairs. Ifirstly, the website API gets the info and makes a HTTP request to cloud website. Then cloud REST full API challenge the stored information as a response to urge request therefore all the mandatory details are shown in cloud website.

\section{A. Modules Explanation}

In this project there are three modules

\section{i. Interfacing with RFID reader}

In this the RFIDreader has been interfaced with Ardunio by use of UART communication. UART or Universal Asynchronous Receiver Transmitter is a dedicated hardware associated with serial communication. The hardware for UART can be a circuit integrated on the microcontroller or a dedicated IC. This is contrast to SPI or I2C, which are just communication protocols. so the rfid reader attach with arduino by this uart vommunication protocol. While rfid reader sense the card it will transmit 12 digit number through UART. Then the Controller will collect that 12 digit number and passess the information to the cloud.

\section{ii. Interfacing with esp8266}

The ESP8266 is a low-cost Wi-Fi microchip, with a full TCP/IP stack and microcontroller capability, produced by Espressif. This small module allows microcontrollers to connect to a Wi-Fi network and make simple TCP/IP connections using Hayes-style commands. we using this low-cost wifi in our project. This esp8266 is being used to connect with the microcontroller which has the data from the hardware and it is transmitted to the cloud with the help of this wifi module. It transmit the data at the rate of 9600 bits per second. We use WIFI connection to transmit the data to cloud.

\section{iii. Creating database in the cloud}

An database will be created for each and individual trolly system, by use of mysql. While user pick the trolly automatically the trolly and the cloud will be connected through WIFI. The product details and cost of the product will be stored in cloud and php web page is created to view the product. the cloud aggregate the data and draw insites from the data for our cloud is used to store details of registered customer, product details, smart card details, shop details. The cloud storage involves atleast one data server that a user connects to via the internet. The custumers send the product details via RFID through the internet to the data server which forwards the information to multiple servers the store data is then accessible through web based interface. cloud storage system involves vast numbers of data server to ensure their availability. That way, if one server requires maintance or fails, the user can rest assure that the day data as been replicated elsewhere to ensure availability. once the purchasing of the products is over the buzzer is being pressed and the payment details or generated to the customers and shopkeepers device through wifi. The data being collected and it is transmitted through cloud to the server and it gives the user access for the payment through mobile. Each trolley has been designed to database it stores the purchased details create to view the product.

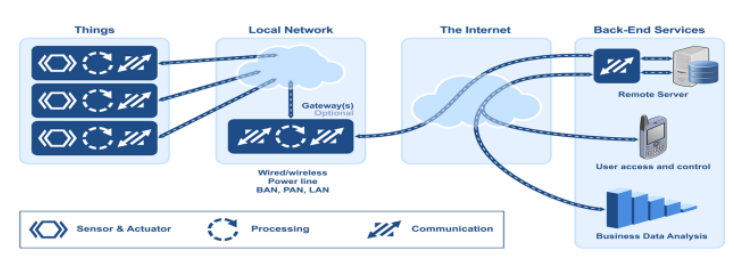

B. Programming the arduino Microcontroller:

The microcontroller of the Smart Cart device requires programming so as to perform given tasks. this is often accomplished using Arduino IDE 1.6.7 software provided within the Arduino website. a well-liked programing language called $\mathrm{C}$ was utilized in the Arduino IDE. Every Arduino program includes two functions: $\operatorname{setup}()$ and $\operatorname{loop}()$. In $\operatorname{setup}()$ function, all global variables are initialized and every one external devices are turned on and initialized, since this function runs only 1 time during the startup of the Arduino. Moreover, some once performed tasks also are put into the setup() function. Therefore, all communications with the Wi-Fi, RFID reader, display modules are initialized and founded within the $\operatorname{setup}()$ function. On the opposite hand, loop() function contains commands or other nested functions that are repeated until Arduino is transitioned or restarted. Therefore, all major tasks are placed in there. In loop function, firstly, Arduino checks the Wi-Fi connection, connection to the Cloud server and connects if unconnected. Then, Arduino requires the user to authenticate and checks the ID card information by sending HTTP GET request to the Cloud server and obtaining user information. After that, Arduino starts reading every RFID tag swiped over the RFID 


\section{International Journal of Engineering Applied Sciences and Technology, 2020 \\ Vol. 4, Issue 11, ISSN No. 2455-2143, Pages 474-477 \\ Published Online March 2020 in IJEAST (http://www.ijeast.com)}

reader and exchange information with the Cloud server.

\section{CONCLUSION}

This develop a shopping cart which can be applied for supermarkets and malls the automated billing facility can avoid queue in the check-out process so that the better shopping experience for customers can be created. Finally, through the recorded data which links between customer and shopping behaviour the proposed system demonstrate the highly potential capability to be integrated into the supermarkets and malls which can also helps the customer to view their billing at anytime anywhere with the help of cloud that stores the information.

\section{REFERENCE}

[1] J. Sales, J. V. Marti, R. Marin, E. Cervera, and P. J. Sanz, "CompaRob: The shopping cart assistance robot," Int. J. Distributed Sensors Networks, vol. 2016, Article ID 4781280, 15 pages, 2016.

[2]RupaliSawant, Kripa Krishnan, ShwetaBhokre, PriyankaBhosale, The RFID Based Smart Shopping Cart, International Journal of Engineering Research and General Science Volume3,Issue 2, March-April, 2015 ISSN 209 1-2730.

[3]Kalyani Dawkhar, Shraddha Dhomase, Samruddhi Mahabaleshwarkar, "Electronic Shopping Cart For Effective Shopping based on RFID", International journal of innovative research in electrical, electronics, instrumentation and management engineering vol. 3, issue 1, Jan 2015.

[4]GalandeJayshree,RutujaGholap, PreetiYadav, RFID Based Automatic Billing Trolley, International Journal of Emerging Technology and Advanced Engineering Website: www.ijetae.com (ISSN 2250-2459, ISO 9001:2008 Certified Journal, Volume 4, Issue 3, March 20 14). C.H.Bill, S.M.Rebecca and M.Yana "Item-Level RFID: Future Direction - Current Status", 2008.

[5]. Raju Kumar, K. Gopalakrishna, K. Ramesha on "Intelligent Shopping Cart" in International Journal of Engineering Science and Innovative Technology (IJESIT) Volume 2, Issue 4, July 2013.

[6]. Zeeshan Ali, Reena Sonkusare.," RFID based Smart Shopping: An Overview.", International Conference on Advances in Communication and Computing Technologies [2014].

[7]. Vrinda Gupta, Niharika Garg, Analytical Model for Automating Purchases using RFID- enabled Shelf and Cart, International Journal of Information and Computation Technology. ISSN0974-2239 Volume 4, NumberS (20 14).

[8].Ashmeet Kaur, Avni Garg, Abhishek Verma, Akshay Bansal, Arvinder Singh, Arduino Based Smart Cart, International Journal of Advanced
Research in Computer Engineering \& Technology (IJARCET) Volume 2, Issue 12, December 2013. [9]. C.H.Bill, S.M.Rebecca and M.Yana "ItemLevel RFID: Future Direction - Current Status", 2008.

[10]. El Mahboul, Abdelaziz, "Smart shopping cart system: a new innovation for Grocery Industry." Turku University of Applied Sciences [2014].

[11]. Karmouche, Amine, and S.AYassine. "Aislelevel scanning for pervasive RFID-based shopping applications." Computer Systems and Industrial Informatics (ICCSII), 2012 International Conference on. IEEE, 2012.

[13]. Bhagyashree Bhumkar, Tejasvini Changal, Bhagyashri Dahifale, Ganesh Deshmukh "Automatic Billing Trolley using RFID and Zigbee with Android Application 2016.

[14]. Ankush Yewatkar, Faiz Inamdar, Raj Singh, Ayushya, Anmol Bandal, "Smart Cart with Automatic Billing, Product Information, Product Recommendation Using RFID \& Zigbee with AntiTheft.", 7th International Conference on Communication, Computing and Virtualization 2016.

[15]. Prassidhi.K and DhanaShri.h.gawali 2017 developed "Innovative Shopping Cart For Smart Cities 